\title{
Even One-Dimensional Mobility Increases Ad Hoc Wireless Capacity
}

\author{
S N. Diggavi * \\ M. Grossglauser ${ }^{\dagger}$ \\ D N C. Tse
}

\begin{abstract}
We study the capacity of ad hoc wireless networks with mobile nodes. The mobility model examined is one where the nodes are restricted to move along one-dimensional paths. We examine the scaling laws for the per-user throughput achievable over long time-scales, making this suitable for applications with loose delay constraints. We show that under this regime of restricted mobility, we attain a constant throughput (i.e., $\Theta(1)$ ) per user, which is significantly higher than the throughput of fixed networks, which decays as $O\left(\frac{1}{\sqrt{n}}\right)$ with the number of nodes $n$, as shown in [4].
\end{abstract}

\section{Introduction}

The study of the capacity of wireless ad hoc networks has received significant attention recently. The seminal work of Gupta and Kumar [4] considered a model in which $n$ nodes are randomly located in a disk of unit area and each node has a random destination node it wants to communicate to. They showed that as the number of nodes $n$ increases, the throughput per source and destination (S-D) pair goes to zero like ${ }^{1} O\left(\frac{1}{\sqrt{n}}\right)$ even allowing optimal scheduling and relaying of packets. The nodes are however assumed to be fixed throughout the duration of the communication sessions. Grossglauser and Tse [3] considered an alternative model in which the nodes are mobile, and they showed that in sharp contrast to the fixed node case, the throughput per S-D pair can actually be kept constant even as the number of nodes scale.

In the mobility model considered in [3], the trajectory of each node is an independent, stationary and ergodic random process with a uniform stationary distribution on the unit disk. This mobility model is a generous one since, (a) it is homogeneous, i.e., every node has the same mobility process, and (b) the sample path of each node "fills the space over time". A natural question that arises

\footnotetext{
*Contact author, School of Computer and Communication Sciences (I\&C), EPFL, Lausanne, Switzerland. Email: suhas.diggavi@epfl.ch

${ }^{\dagger}$ School of Computer and Communication Sciences (I\&C), EPFL, Lausanne, Switzerland. Email: matthias.grossglauser@epfl.ch

${ }^{\ddagger}$ Department of of EECS, UC Berkeley, Berkeley, California, USA. Email: dtse@eecs.berkeley.edu

${ }^{1}$ We use the notation $f(n)=\Theta(g(n))$ to denote $f(n)=O(g(n))$ as well as $g(n)=O(f(n))$. Here $f(n)=O(g(n))$ means $\lim \sup _{n \rightarrow \infty}\left|\frac{f(n)}{g(n)}\right|<\infty$.
} 
is then how strongly the throughput result in [3] depends on these two features of the mobility model. This is the main theme of this paper, where we try to isolate some of the reasons why we get a constant throughput by studying a more restricted mobility model.

We show in this paper that the throughput result in [3] still holds even when nodes have much more limited mobility patterns. Specifically, the mobility model discussed in this paper is an abstraction that embodies two salient features that many real mobility processes seem to possess (e.g., cars traveling on roads, people walking in buildings or cities, trains, satellites circling earth), which are not captured by the model in [3]. First, an individual node typically visits only a small portion of the entire space, and rarely leaves this preferred region. Second, the nodes do move frequently within their preferred regions, and an individual region often covers a large distance.

As an extreme abstraction of such mobility processes, we study mobility patterns where nodes move along a given set of one-dimensional paths. In order to isolate the effects of one-dimensional mobility from edge effects, we consider a model in which the nodes are on a unit-radius sphere but each node is constrained to move on a single-dimensional great circle. These great circles are random, but remain fixed over time. Each node moves randomly along its own circle, independently of the other nodes. The mobility process is therefore ergodic only in a single dimension. There is another reason to study mobility over great circles of a sphere instead of line segments or curves in two-dimensional spaces. Many of the results derived on the surface of the sphere can be translated to one-dimensional paths on a plane using an inversion mapping from a sphere to a plane as used in Section IV in [4]. In fact all the results in the random model used in [4] were actually proved on the surface of a sphere and were translated to the unit area on a plane using such an argument. Therefore, the insights gained from studying our one-dimensional mobility model can be used in paths over planes as well.

In the mobility model considered in [3], the distribution of the random distance between two nodes was the same for every pair. In the present model, as every node is constrained to its great circle, the distance distribution depends on the node pair. Thus, the homogeneity assumption in [3] is now relaxed. In particular, there can be pairs of nodes that are far more likely to be in close proximity to each other than other pairs. For example, if two great circles nearly overlap, the probability of close encounter between the nodes is significantly larger than for two great circles that intersect at a large angle. This lack of homogeneity implies, as shown in Section 4.2, that we can construct configurations where constant throughput is unattainable even with mobility. Given this, it is now unclear what the throughput scaling law that can be attained by the one-dimensional mobility model considered in the paper. Therefore, this also focuses our attention on what property in the mobility pattern allows us to attain a constant throughput, i.e., sufficient conditions which allows $\Theta(1)$ throughput.

Since the capacity of such a network then depends on the constellation of the great circles the question becomes one of scaling laws for a random configuration. One of the key contributions of our work in this paper is the identification of "typical" configurations, on which the average long-term throughput per node can be kept constant as the number of nodes increases. Therefore, even for a particular deterministically chosen configuration which satisfies the typicality condition, the result continues to hold. Our main result is that if the locations of the great circles are chosen randomly and independently, then for almost all constellations of such great circles, the throughput per S-D pair can be kept constant as the number of nodes increase. Therefore, for random configurations 
the probability of an "atypical" configuration is shown to go to zero asymptotically in network size. Thus, although each node is restricted to move in a one-dimensional space, the same asymptotic performance is achieved as in the case when they can move in the entire 2-D region. Intuitively the definition of typical configuration, defined formally in Section 4.2, is interpreted as configurations where the fraction of great circles intersecting any given area is close to its expected number. That is, the empirical probability counts are close to the underlying probability of a random great circle intersecting that area. As mentioned earlier, we focus on the unit-radius sphere instead of the unit disk to avoid edge effects, but the results should also be valid for nodes moving along line segments within the unit disk.

The paper is organized as follows. We introduce the model under consideration and the definition of per-user throughput in Section 2. In Section 3 we state the protocol studied and the main result of the paper. The ingredients of the proof are developed in Section 4 and some of the details of the proofs are given in the Appendices. Finally in Section 5 we conclude with a discussion of the scope of our result and some of the open questions.

\section{Model and Performance Measure}

The mobility patterns of the users are defined by a constellation $\mathcal{C}=\left\{G_{1}, \ldots, G_{n}\right\}$ of great circles on the unit-radius sphere. Generate a point uniformly randomly on the sphere, and the great circle is taken as the "equator" corresponding to this point as the pole, i.e., the great circle associated with $y$ is in the plane perpendicular to $y$ and the center of the sphere. We generate $n$ such independent great circles $\left\{G_{1}, \ldots, G_{n}\right\}$ to form a random constellation $\mathcal{C}$.

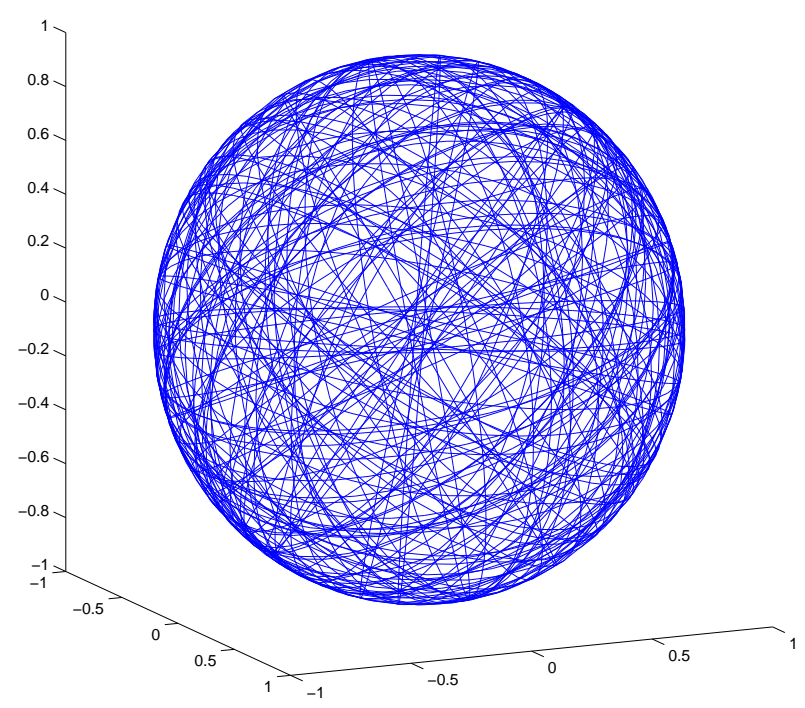

Figure 1: A sample constellation with $n=100$ random great circles. In our model, each node is restricted to move on its great circle.

Over time, each node moves in a stationary, ergodic manner along its own circle with a uniform 
stationary distribution. The mobility processes of the nodes are identically distributed and independent of each other. Since the movements of the nodes are independent of each other we do not expect any "synchronization" that might occur in the mobility pattern across nodes.

Other than the mobility pattern of the nodes, the rest of the model is the same as the one used in [3], which we briefly review here. Each of the $n$ nodes is a source node for one communication session, and a destination node for another session. The S-D pairs are randomly selected and independent of the constellation. There are $n$ communication sessions in parallel.

At (slotted) time $t$, a packet is successfully transmitted from node $i$ to node $j$ if the signal-tointerference ratio (SIR) exceeds a target prescribed requirement $\beta$. The channel attenuation of the signal from node $i$ to node $j$ is given by $\frac{1}{r^{\alpha}}$ where $r$ is the distance ${ }^{2}$ between the two nodes and $\alpha$ is a parameter greater than 2. This models large-scale path loss characteristics of the channel. Packets can be transmitted directly from a source to its destination, or they can go through one or more other nodes serving as relays.

Consider a scheduling and relay policy $\pi$. Let $M_{i}^{\pi}(t)$ be the number of source node $i$ packets (either 0 or 1 ) that destination $d(i)$ receives at time $t$ under policy $\pi$. In a system of size $n$, we shall say a policy $\pi$ achieves a throughput $\lambda(n)$ if for every $\mathrm{S}-\mathrm{D}$ pair $i$,

$$
\liminf _{T \rightarrow \infty} \frac{1}{T} \sum_{t=1}^{T} M_{i}^{\pi}(t) \geq \lambda(n) .
$$

We say that a policy achieves a throughput $\lambda>0$, if asymptotically a constant throughput is achievable, i.e.,

$$
\lim _{n \rightarrow \infty} \operatorname{Pr}\{\lambda(n) \geq \lambda \text { is feasible }\}=1 .
$$

Note that the performance of a policy depends implicitly on the constellation $\mathcal{C}$ of great circle.

\section{Main Result}

In [3] a simple policy $\pi$ is shown to achieve asymptotically a constant throughput. Let us first review the policy.

Protocol 3.1 There are two phases, alternating in time. In both phase nodes are randomly divided into $n / 2$ senders and $n / 2$ receivers at every time slot. At each time, every node carries source packets, which originate from that node, and relay packets, which originated from other nodes and to be forwarded to their final destinations. In phase 1, each sender attempts to transmit a source packet to its nearest receiver, who will serve as a relay for that packet In phase 2, each sender identifies its nearest receiver and attempts to transmit a relay packet destined for it, if the sender has one. When nodes transmit, they all use the same power level.

\footnotetext{
${ }^{2}$ Throughout this paper the distances are measured on the surface of the disk. This is defined as the length of the segment of a great circle connecting any two points on the surface of the sphere $\mathbb{S}[5]$.
} 
Basically, the first phase spreads the packets from each source node to every other node in the network, and the second phase merges the packets to the destination nodes. See Figure 2. When the nodes are moving in a stationary ergodic manner, uniformly on the disk, packets are spread to the fullest extent and each node carries the packets for every S-D pair in the steady-state. It was shown that this state of affairs results in an asymptotically constant throughput per S-D pair.

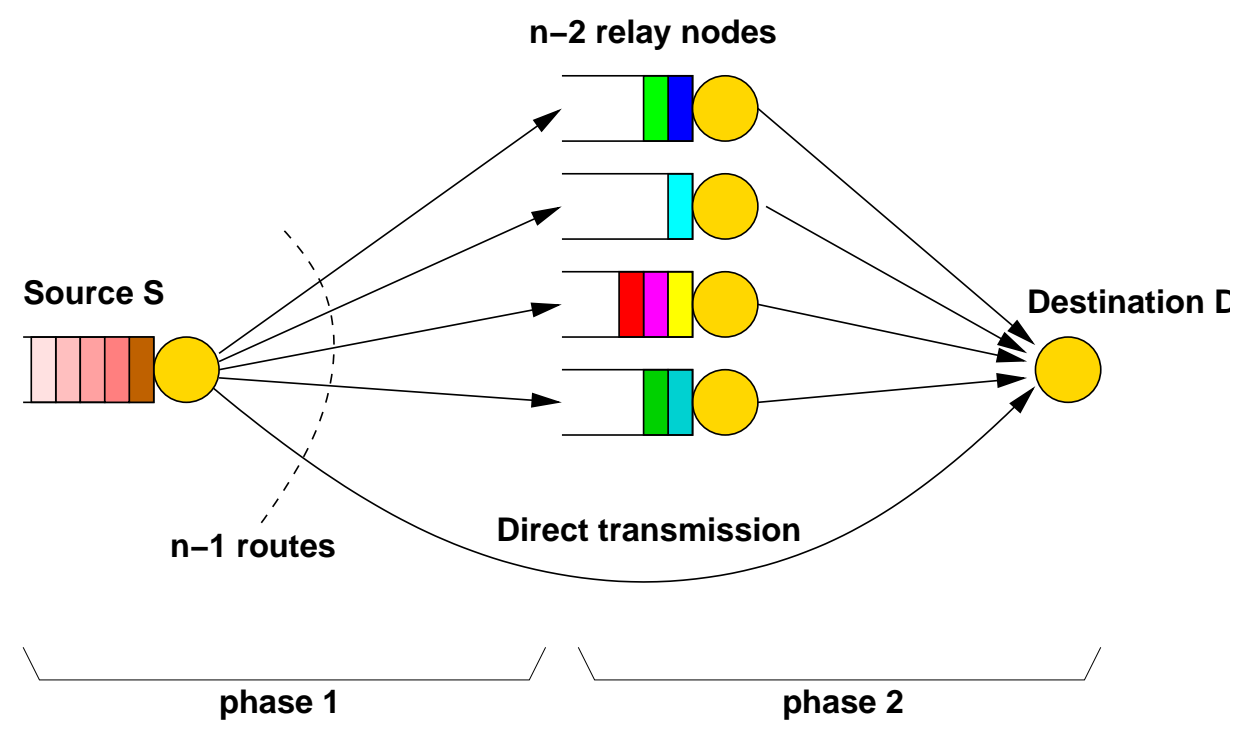

Figure 2: A source node spreads its packets to $n-2$ relay nodes in phase 1 , and the packets are merged back into the destination node in phase 2. There is also direct transmission between the source and the destination when they are close to each other.

The main quest in this paper is to determine what happens when each node is constrained to move on a one-dimensional great circle. The performance of the policy clearly depends on the constellation $\mathcal{C}$ of the great circles. It is in fact not true that the policy can achieve a constant throughput for every possible constellation, but only for most.

Our main result is as follows.

Theorem 3.2 There exists a $\lambda>0$ such that the above policy $\pi$ achieves a throughput of $\lambda$ for almost all constellations as $n \rightarrow \infty$, i.e. the probability of the set of constellations for which the policy achieves a throughput of $\lambda$ goes to 1 as $n \rightarrow \infty$. 


\section{Main Ideas in the Proof}

\section{Notation}

$\begin{array}{ll}n & \text { the number of nodes/great circles } \\ i, j & \text { sender and receiver of an individual transmission } \\ S, D & \text { the source and destination of a session } \\ \pi & \text { scheduling policy } \\ \lambda & \text { achievable throughput } \\ M_{S}^{\pi}(r) & \text { average throughput from } S \text { to } D(S) \text { under policy } \pi \\ M_{i \rightarrow j}^{\pi} & \text { long-term average of successful transmission from } i \text { to } j \text { under } \pi \\ \alpha & \text { the path loss exponent } \\ \beta & \text { the SIR threshold for a successful transmission } \\ \mathbb{S} & \text { the surface of the unit-radius } 3 \text {-dimensional sphere } \\ X_{i}(t) & \text { the position of node } i \text { at time } t \\ D_{x, r} & \text { a disk centered at } x \text { of radius } r \text { on the unit-radius sphere } \\ V C_{d}(.) & \text { the Vapnik-Chervonenkis dimension of a set } \\ G & \text { a great circle } \\ \mathcal{C} & \text { a constellation of great circles, } i . e ., \text { a set of } n \text { great circles } \\ M_{\mathcal{C}}\left(D_{x, r}\right) & \text { the number of great circles intersecting } D_{x, r} \\ \bar{M}\left(D_{x, r}\right) & \text { expected number of great circles intersecting } D_{x, r} \\ N_{\mathcal{C}}\left(x, r_{1}, r_{2}\right) & \text { the number of great circles intersecting the annulus } D_{x, r_{2}} \backslash D_{x, r_{1}} \\ N_{k} & \text { abbreviation for } N_{\mathcal{C}}\left(x, d_{k-1}, d_{k}\right), i . e . \text { number of great circles in the annulus } D_{x, d_{k}} \backslash D_{x, d_{k-1}} \\ d_{\mathbb{S}}(x, y) & \text { the distance of a points } x \text { and } y \text { on the sphere } \\ d(x, G) & \text { the distance of a point } x \text { from a great circle } G, i . e . d(x, G)=\inf { }_{y \in G} d_{\mathbb{S}}(x, y) \\ \gamma & \text { normalized number of disks for binning } \\ d_{k} & \text { tor } k=1, \ldots, \gamma \sqrt{n}, \text { set of distances such that sin }\left(d_{k}\right)=\frac{k}{\gamma \sqrt{n}} \\ \mathcal{S}_{t}, \mathcal{R}_{t} & \text { the sets of potential senders and potential receivers at time } t \\ j(i) & \text { the closest } j \in \mathcal{R}_{t} \text { of } i \in \mathcal{S}_{t} \\ P_{i} & \text { the received signal power at the receiver } j(i) \\ I_{i} & \end{array}$

\subsection{Key Properties to Guarantee High Throughput}

A closer examination of [3] reveals that there are two main reasons why the policy $\pi$ achieves an asymptotically constant throughput in the mobility model considered there:

- Property I: Every node spends the same order of time as the nearest neighbor to every other node. This ensures that each source can spread its packets uniformly across all other nodes, all acting as relays, and these packets can in turn be merged back into their respective final destinations.

- Property II: When communicating with the nearest neighbor receiver, the capture probability is not vanishingly small even in a large system, even though there are $O(n)$ interfering 
nodes transmitting simultaneously.

The first property ensures that there are no bottlenecks/hotspots in the relaying mechanism. It ensures that if we think of the different relays as queues for the S-D pair (see Figure 2), there is an equitable distribution of the traffic over these queues.

The second property is due to the heavy-tailed characteristic of the received power distribution from a random interfering node: the complementary cumulative distribution function (ccdf) decays faster than $x^{-\frac{2}{\alpha}}$. This ensures that the received power from the nearest neighbor is of the same order as the total interference from the other senders, so that the probability that the SIR exceeds the target threshold does not vanish with $n$. The decay condition in turn depends on the fact that the nodes are uniformly distributed in the space.

\subsection{Typical Constellations}

Going back to our present mobility model, it is not difficult to see that these two key properties do not necessarily hold for any arbitrary constellation $\mathcal{C}$ of great circles. For example, consider a degenerate constellation with only two distinct great circles, where half of the nodes live on one circle and the other half live on the other. Since there are $n / 2$ nodes on each great circle, the typical distance between nearest neighbors is of order $1 / n$. It can be seen that a given node $i$ is the nearest neighbor to a node on the same great circle for a fraction of order $1 / n$ of the time, while it is the nearest neighbor to a node on the other great circle only for a fraction of order $1 / n^{2}$ of the time (when both are of distance $1 / n$ to one of the intersections of the two circles.) Thus, property I does not hold, and the policy cannot deliver an $O(1)$ throughput for any S-D pair whose source and destination do not lie on the same great circle. Intuitively, the two intersections of the circles become bottlenecks through which too much of the traffic has to pass.

The proof of Theorem 3.2 shows that for typical constellations, property I and II hold. The key technical step is an appropriate definition of "typicality". Basically, we require that for any point $x$ on the sphere $\mathbb{S}$, there are not an "atypically" large number of great circles "close" to it. More precisely, for a given constellation $\mathcal{C}$, define $M_{\mathcal{C}}\left(D_{x, r}\right)$ to be the number ${ }^{3}$ of great circles passing through a disk $D_{x, r}$ of radius $r$ around $x \in \mathbb{S}$. Let $\bar{M}\left(D_{x, r}\right)$ be the expected number of such great circles in a random constellation. Therefore intuitively it means that a typical configurations is one whose empirical distribution of the number of great circles in any given area on the surface of the sphere is close to the underlying probability generating the configuration.

Definition 4.1 A constellation $\mathcal{C}$ is said to be $\epsilon$-typical if

$$
\frac{\left|M_{\mathcal{C}}\left(D_{x, r}\right)-\bar{M}\left(D_{x, r}\right)\right|}{n}<\epsilon \quad \text { for every point } x \in \mathbb{S} \text { and every } r>0 .
$$

\footnotetext{
${ }^{3} M_{C}\left(D_{x, r}\right)=\sum_{i=1}^{n} \mathbf{1}_{\left\{\text {great circle } G_{i} \text { intersecting } D_{x, r}\right\}}$, where $\mathbf{1}_{\{\cdot\}}$ denotes the indicator function.
} 
Lemma 4.2 For any $\epsilon, \delta>0$, the probability that a constellation $\mathcal{C}$ is $\epsilon$-atypical is less than $\delta$ provided that the system size $n>K(\epsilon, \delta)$, where

$$
K(\epsilon, \delta):=\max \left\{\frac{V C_{d}(\mathcal{D})}{\epsilon} \log \frac{16 e}{\epsilon}, \frac{4}{\epsilon} \log \frac{2}{\delta}\right\}
$$

Proof: To prove the lemma, we need to show that the empirical measure on the number of randomly generated great circles intersecting a disk $D_{x, r}$ converges uniformly (on the set of all disks $\mathcal{D}$ ) to the probability of a randomly generated great circle intersecting the disk. This involves uniform convergence of this empirical probability measure and uses the Vapnik-Chervonenkis Theorem [1]. To do this we use some results from Gupta and Kumar [4] and we use the notation from that paper. Lemma 4.11 (pp 400, [4]) states that a great circle intersects a disk if and only if the polar point associated with the great circle is contained in the equatorial band of the disk (see Figure 5 of that paper). The empirical measure $M_{\mathcal{C}}\left(D_{x, r}\right)$ is therefore the number of randomly generated points on the sphere that lie in the equatorial band corresponding to the disk. The key parameter governing the uniform convergence of the empirical measure is the $\mathrm{VC}$ dimension of the set of equatorial bands corresponding to the set of all disks on the sphere. From Lemma 4.12 of [4], the VC dimension of this set is finite, which implies the uniform convergence of the empirical measure of number of great circles over the set of disks on the sphere.

More specifically:

$$
\begin{aligned}
& \operatorname{Pr}\left\{\sup _{D \in \mathcal{D}}\left|\frac{M_{\mathcal{C}}(D)}{n}-\operatorname{Pr}\{G \cap D \neq \emptyset\}\right|>\epsilon\right\}<\delta \\
& \text { for } n>\max \left\{\frac{V C_{d}(\mathcal{D})}{\epsilon} \log \frac{16 e}{\epsilon}, \frac{4}{\epsilon} \log \frac{2}{\delta}\right\} \stackrel{\text { def }}{=} K(\epsilon, \delta),
\end{aligned}
$$

where $\mathcal{D}$ is the set of all disks on the sphere and it is shown by [4] (Lemma 4.7, pp 398) that this has finite VC-dimension.

The number $\bar{M}(D(x, r))$ can be explicitly computed.

$$
\bar{M}\left(D_{x, r}\right)=\mathrm{E}\left[M_{C}\left(D_{x, r}\right)\right]=\sum_{i=1}^{n} \operatorname{Pr}\left\{G_{i}: d\left(x, G_{i}\right)<r\right\} \stackrel{(a)}{=} n \operatorname{Pr}\{G: d(x, G)<r\} .
$$

Here $(a)$ is because $\left\{G_{1}, \ldots, G_{n}\right\}$ are independently and identically distributed.

The probability of a randomly generated great circle intersecting a disk is the probability that a randomly generated point on the sphere is in the equatorial band corresponding to the disk. If the area of the equatorial band of a disk $D_{x, r}$ is denoted by $E\left(D_{x, r}\right)$, then

$$
E\left(D_{x, r}\right)=4 \pi \sin (r)
$$

on a unit-radius sphere. Therefore, the probability of a randomly generated great circle to intersect a disk $D_{x, r}$ is

$$
\operatorname{Pr}\{G: d(x, G)<r\}=\sin (r)
$$


Hence,

$$
\bar{M}\left(D_{x, r}\right) / n=\sin (r)
$$

We now apply Lemma 4.2 to a particular set of disks. As will become apparent later, we are interested in $\lceil\gamma \sqrt{n}\rceil$ disks around a point $x \in \mathbb{S}$ with radii, $d_{i}, i=1, \ldots,\lceil\gamma \sqrt{n}\rceil$, such that

$$
\sin \left(d_{i}\right)=\frac{i}{\lceil\gamma \sqrt{n}\rceil}
$$

For brevity of notation, we will interchangeably use $\lceil\gamma \sqrt{n}\rceil$ with $\gamma \sqrt{n}$ with the understanding that this would not make a difference in the asymptotics. Also note that $\gamma$ is a constant parameter that controls the number of disks and is used for analysis. For this set of disks (which depend on $n$ ), we get the following result,

$$
\begin{aligned}
& \operatorname{Pr}\left\{\sup _{\substack{1 \leq i \leq \gamma \sqrt{n} \\
x \in \mathbb{S}}} \frac{1}{n}\left|M_{\mathcal{C}}\left(D_{x, d_{i}}\right)-\bar{M}\left(D_{x, d_{i}}\right)\right|>\epsilon_{n} / 2\right\}<\delta_{n} / 2 \\
& \text { for } n>\max \left\{\frac{2 V C_{d}(\mathcal{D})}{\epsilon_{n}} \log \frac{32 e}{\epsilon_{n}}, \frac{8}{\epsilon_{n}} \log \frac{4}{\delta_{n}}\right\},
\end{aligned}
$$

An important consequence of this is that we can analyze the number of great circles which intersect an annulus, i.e., for $r_{1}<r_{2}$,

$$
N_{\mathcal{C}}\left(x, r_{1}, r_{2}\right) \stackrel{\text { def }}{=} M_{\mathcal{C}}\left(D_{x, r_{2}}\right)-M_{\mathcal{C}}\left(D_{x, r_{1}}\right) .
$$

In analogy to (6), we define

$$
\bar{N}\left(x, r_{1}, r_{2}\right)=\mathrm{E}\left[N_{\mathcal{C}}\left(x, r_{1}, r_{2}\right)\right]=\bar{M}\left(D_{x, r_{2}}\right)-\bar{M}\left(D_{x, r_{1}}\right) .
$$

Using (5) and the union bound, we get:

$$
\operatorname{Pr}\left\{\sup _{1 \leq i \leq \gamma \sqrt{n}} \frac{1}{n}\left|N_{\mathcal{C}}\left(x, d_{i-1}, d_{i}\right)-\bar{N}\left(x, d_{i-1}, d_{i}\right)\right|>\epsilon_{n}\right\} \leq \delta_{n}
$$

for $n>K\left(\epsilon_{n}, \delta_{n}\right)$.

Note that we can choose $\epsilon_{n}=\frac{1}{2 \gamma \sqrt{n}}, \delta_{n}=\frac{1}{n^{2}}$ in the above result. For this choice, together with the observation that the expected number of great circles intersecting each annulus is $\Theta(\sqrt{n})$, implies:

$$
N_{i} \stackrel{\text { def }}{=} N_{\mathcal{C}}\left(x, d_{i-1}, d_{i}\right)=\Theta(\sqrt{n}),
$$

with high probability.

\subsection{Throughput Analysis on Typical Constellation}

Our proof strategy is to show that Properties I and II hold for $\Theta\left(\frac{1}{\sqrt{n}}\right)$-typical constellations, so that the throughput achievable by Protocol 3.1 is high. This is given respectively by the following 
two lemmas. We will be assuming the constellation $\mathcal{C}$ is $\Theta\left(\frac{1}{\sqrt{n}}\right)$-typical and considered fixed. The randomness to which the probabilities refer is due to the random positions of the nodes in their respective great circles, and the random partition of the set of nodes into potential senders and receivers in each time slot.

The following lemma formalizes Property I.

Lemma 4.3 There exist constant $c_{1}>0$ and integer $n_{0}$ such that for any two arbitrary nodes $i$ and $j$, the probability that node $j$ is the nearest neighbor of node $i$ is at least $c_{1} / n$, provided $n>n_{0}$.

Proof: We will lower bound the probability that $j$ is the nearest neighbor of $i$ with the probability that $j$ is within a disk of radius $d_{1}=\sin ^{-1}\left(\frac{1}{\sqrt{n}}\right)$ centered around $i$ and that there is no other node in such a disk. More precisely, consider a disk $D_{X_{i}, d_{1}}$ of radius $d_{1}$ around the location $X_{i}$ of sender $i$.

$$
\operatorname{Pr}\{j \text { is closest node to } i\} \geq \operatorname{Pr}\left\{X_{j} \in D_{X_{i}, d_{1}}\right\} \operatorname{Pr}\left\{\text { No other node is in } D_{X_{i}, d_{1}}\right\} .
$$

Let the intersection point between the great circles $G_{i}, G_{j}$ corresponding to $i$ and $j$ be $F(i, j) \in \mathbb{S}$ (see Figure 3).

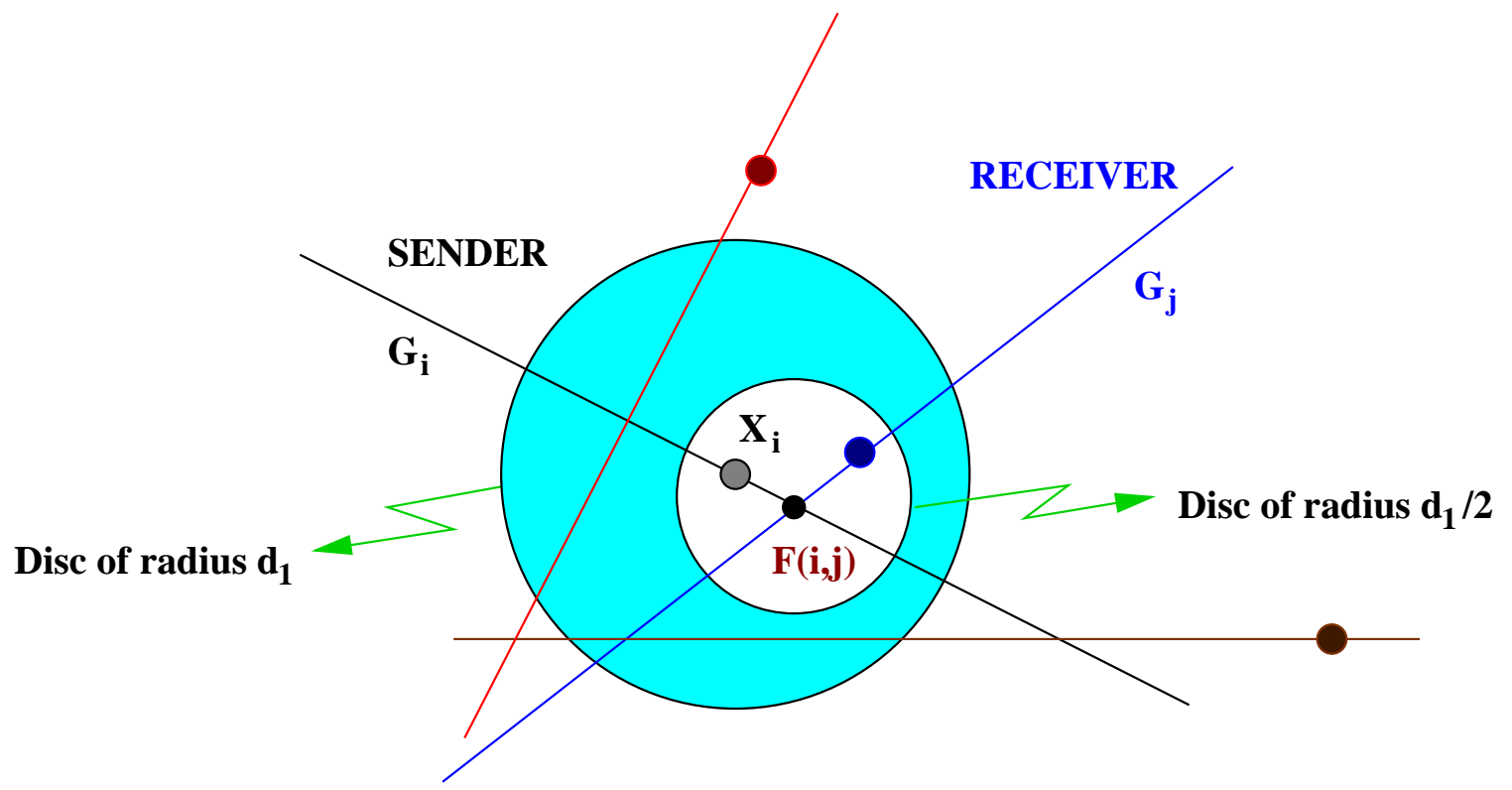

Figure 3: Region of intersection between two great circles $G_{i}, G_{j}$ used in the proof of Lemma 4.3

We can see that

$$
\begin{aligned}
& \operatorname{Pr}\left\{X_{j} \in D_{X_{i}, d_{1}}\right\} \geq \operatorname{Pr}\left\{X_{j} \in D_{F(i, j), \frac{d_{1}}{2}}\right\} \operatorname{Pr}\left\{X_{i} \in D_{F(i, j), \frac{d_{1}}{2}}\right\}=\left(\frac{d_{1}}{2 \pi}\right)^{2} \\
& \operatorname{Pr}\left\{\text { No other node is in } D_{X_{i}, d_{1}}\right\} \geq\left(1-\frac{2 d_{1}}{2 \pi}\right)^{N_{1}},
\end{aligned}
$$

where $N_{1}$ is the number of great circles passing through the disk $D_{X_{i}, d_{1}}$, which we know by the typicality of the constellation is $N_{1}=\Theta(\sqrt{n})$. Hence, using these results and using binning parameter 
$\gamma=1$ we get,

$\liminf _{n \rightarrow \infty} n \operatorname{Pr}\{j$ is closest receiver to $i\} \geq \liminf _{n \rightarrow \infty}\left[\sqrt{n} \sin ^{-1}\left(\frac{1}{\sqrt{n}}\right) \frac{1}{2 \pi}\right]^{2}\left(1-\frac{1}{\pi} \sin ^{-1}\left(\frac{1}{\sqrt{n}}\right)\right)^{N_{1}}=c_{1}>0$.

Hence there exists $n_{0}$ such that for $n>n_{0}$, the probability that node $j$ is the nearest neighbor of node $i$ is at least $c_{1} / n$.

Basically, the proof of the above lemma says that any two nodes are nearest neighbors to each other typically when they are both of order $1 / \sqrt{n}$ from the intersection of their great circle and that the probability with which this occurs is $O\left(\frac{1}{n}\right)$.

The following lemma formalizes Property II.

Lemma 4.4 Fix a given time slot. For an arbitrary sender node $i$, let $P_{i}$ be the received power of its signal at the intended receiving node, and let $I_{i}$ be the total power of the interference at that receiver, due to all the other senders. Then there exists constant $c_{2}>0$ such that the signal-to-interference ratio at the receiver satisfies

$$
\liminf _{n \rightarrow \infty} \operatorname{Pr}\left\{\frac{P_{i}}{I_{i}}>\beta\right\}>c_{2}
$$

Let $j(i)$ denote the receiver closest to sender node $i$. Conditional on that receiver's position $X_{j(i)}$, $P_{i}$ and $I_{i}$ are independent. This is because the position $X_{k}$ of every interfering sender $k \in \mathcal{S}_{t}, k \neq i$ is independent of $X_{i}$. However, note that the conditional distribution of $P_{i}$ and $I_{i}$ does depend on $X_{j(1)}$.

The proof of Lemma 4.4 hinges on the following two results.

Lemma 4.5 Fix a time slot. For an arbitrary sender node $i \in \mathcal{S}_{t}$ at location $X_{i}$, for any constant $B>0$ there exists a constant $c_{P}>0$ such that the received signal power $P_{i}$ at the intended receiver of $i$ satisfies

$$
\liminf _{n \rightarrow \infty} \operatorname{Pr}\left\{P_{i}>B n^{\alpha / 2} \mid X_{i}\right\}>c_{P}
$$

Lemma 4.6 Fix a time slot. For an arbitrary sender node $i \in \mathcal{S}_{t}$ at location $X_{i}$, there exist constants $A>0$ and $c_{I}<1$ such that the received interference power at the intended receiver of $i$ (i.e., $j(i)$ ) at location $X_{j(i)}$ satisfies

$$
\liminf _{n \rightarrow \infty} \operatorname{Pr}\left\{I_{i}>A n^{\alpha / 2} \mid X_{j(i)}\right\}<c_{I}
$$

These two lemmas are proved in Appendix A and B, respectively. We have already seen that nearest neighbors are typically at distance $1 / \sqrt{n}$, so it is not too surprising that the received power $P_{i}$ is of 
the order $n^{\alpha / 2}$, as stated in Lemma 4.5. The other senders are more or less uniformly distributed on the sphere, by the typicality of the constellation. The total interference $I_{i}$ at the receiver is therefore of the same order as a sum of $O(n)$ uniformly distributed senders, calculated in [3] to be of order $n^{\alpha / 2}$. Therefore, the result stated in Lemma 4.6 is not unexpected. However, this intuition is imprecise, and the technical arguments to make a precise statement rely heavily on the typicality of the constellations; the details are given in B.

We are now in a position to prove Lemma 4.4 .

Proof: (Lemma 4.4) We need to show that the probability that for a particular sender the signal-to-interference ratio at its nearest receiver (chosen by the sender as the intended receiver) is large enough, so that a successful transmission is accomplished. Let us define the following events

$$
\begin{aligned}
& E=\left\{P_{i}>\beta A n^{\alpha / 2}\right\} \\
& F=\left\{I_{i}<A n^{\alpha / 2}\right\}
\end{aligned}
$$

From Lemma 4.6 we see that, $\limsup _{n \rightarrow \infty} \operatorname{Pr}\left\{I_{i}>A n^{\alpha / 2} \mid X_{j}\right\}<c_{I}<1$, therefore $\liminf _{n \rightarrow \infty} \operatorname{Pr}\left\{F \mid X_{j}\right\} \geq$ $1-c_{I}>0$. Using this we have,

$$
\begin{aligned}
\operatorname{Pr}\left\{\frac{P_{i}}{I_{i}}>\beta\right\} & =\mathbb{E}_{X_{i}, X_{j(i)}}\left[\operatorname{Pr}\left\{\frac{P_{i}}{I_{i}}>\beta \mid X_{i}, X_{j(i)}\right\}\right] \\
& \geq \mathbb{E}_{X_{i}, X_{j(i)}}\left[\operatorname{Pr}\left\{E \bigcap F \mid X_{i}, X_{j(i)}\right\}\right] \\
& =\mathbb{E}_{X_{i}, X_{j(i)}}\left[\operatorname{Pr}\left\{E \mid X_{i}, X_{j(i)}\right\} \operatorname{Pr}\left\{F \mid E, X_{i}, X_{j(i)}\right\}\right] \\
& =\mathbb{E}_{X_{i}, X_{j(i)}}\left[\operatorname{Pr}\left\{E \mid X_{i}, X_{j(i)}\right\} \operatorname{Pr}\left\{F \mid X_{j(i)}\right\}\right]
\end{aligned}
$$

Hence by taking limits we obtain,

$$
\begin{aligned}
\liminf _{n \rightarrow \infty} \operatorname{Pr}\left\{\frac{P_{i}}{I_{i}}>\beta\right\} & \geq \liminf _{n \rightarrow \infty} \mathbb{E}_{X_{i}, X_{j(i)}}\left[\operatorname{Pr}\left\{E \mid X_{i}, X_{j(i)}\right\} \operatorname{Pr}\left\{F \mid X_{j(i)}\right\}\right] \\
& \stackrel{(a)}{\geq}\left(1-c_{I}\right) \liminf _{n \rightarrow \infty} \mathbb{E}_{X_{i}, X_{j(i)}}\left[\operatorname{Pr}\left\{E \mid X_{i}, X_{j(i)}\right\}\right] \stackrel{(b)}{>} 0
\end{aligned}
$$

where in $(a),(b)$ we have exchanged limits and integrals (possible by the Bounded Convergence Theorem [6]) and obtained the inequalities using Lemmas 4.5-4.6.

This completes the proof of Lemma 4.4.

We are now in a position to prove Theorem 3.2, the main result of the paper.

Proof: (Theorem 3.2) We show that for a $1 / \sqrt{n}$-typical constellation, Protocol 3.1 achieves a throughput $\lambda>0$ for all S-D pairs. If this is done, then the proof is completed by observing that the probability of a $1 / \sqrt{n}$-typical constellation goes to 1 (asymptotically in the system size) due to Lemma 4.2.

The average throughput $M_{S}^{\pi}(r)$ between a source $S$ and its destination $D(S)$ through a relay $r$ is given by

$$
M_{S}^{\pi}(r)=\min \left\{M_{S \rightarrow r}^{\pi}, M_{r \rightarrow D}^{\pi}\right\}
$$

$$
\text { where } M_{i \rightarrow j}^{\pi}=\lim _{T \rightarrow \infty} \sum_{t=1}^{T} \mathbf{1}_{\{\text {successful txmt from } i \text { to } j \text { at time } t\}}
$$


Due to ergodicity of the node mobility processes, $M_{i \rightarrow j}^{\pi}$ is equal to the probability of successful transmission between $i$ and $j$. In order for a successful transmission to occur between the senderreceiver pair $(i, j)$, the $j$ should be the closest receiver to sender $i$, and the signal-to-interference ratio should be above threshold $\beta$. Using Lemma 4.3 we know that for large enough system size $n$, the probability of $j$ being the closest receiver to $i$ is $\Theta\left(\frac{1}{n}\right)$. Lemma 4.4 tells us that the probability that the signal-to-interference threshold is met is bounded away from zero. Putting these two together, the probability of successful transmission between a particular sender-receiver pair $(i, j)$ is $\Theta\left(\frac{1}{n}\right)$. Using this, we have shown that $M_{S}^{\pi}(r)=\Theta\left(\frac{1}{n}\right)$. As there are $\Theta(n)$ such relays, summing the throughput over these relays gives us the result that the average throughput for any $\mathrm{S}-\mathrm{D}$ pair is $O(1)$ for a typical constellation.

\section{Discussion}

In this paper we have shown that the throughput of ad hoc wireless networks can be enhanced even with limited mobility, i.e., when nodes move on great circles. The proof of this result relied on what we called typical constellations. This was important since the throughput depends on the constellation of great circles, and indeed for some configurations the throughput achievable is low. One of the contributions of this work was in isolating precisely a sufficient property for the constellation to yield $\Theta(1)$ throughput per node. The relaying mechanism used for showing an achievable throughput requires that there exist a large number, $\Theta(n)$, of short hops that a source can have to a particular destination. However, as was shown in the example of Section 4.2, even when this property is satisfied, we could have constellations which do not have $\Theta(1)$ throughput. In this example, though the source node did encounter a large number of short routes, there was a larger proclivity for the source to have a relay node from the same great circle rather than one on the alternate circle. Therefore, this created a disjoint "connectivity" graph for successful transmission from source to destination and therefore yielding the negative result for throughput. Another way one can think of "good" constellations is that of uniformly spreading the circles so that there is less clustering. However, this notion can be misleading since a constellation where all the users lie on the same great circle yields $\Theta(1)$ throughput. This shows that the necessary condition for such a result to hold is elusive, and it is important to understand the sufficient condition for a general class of constellations. Hence, we believe that the notion of typicality on constellations defined in this paper is a natural and useful one. This also leads to the question that we are still attempting to answer, i.e., essentially what in the mobility model allows $\Theta(1)$ throughput per user? This paper takes one step in isolating the properties of mobility which allows $\Theta(1)$ throughput, but the general question remains unanswered. 


\section{Appendices}

\section{A Proof of Lemma 4.5}

Proof: To prove this lemma, we focus on the great circles intersecting the innermost disk $D_{X_{i}, d_{1}}$ centered at sender $i$. A sufficient condition for the normalized received power $n^{-\alpha / 2} P_{i}$ to be above a threshold $B$ is that at least one of the nodes on those great circles be close to $X_{i}$ and be in the set $\mathcal{R}_{t}$. More precisely, focus on a great circle $G$ intersecting $D_{X_{i}, d_{1}}$. Let $V$ denote the point on $G$ closest to $X_{i}$, and let $\mathcal{Z}=\left\{U \in G: d(U, V) \leq d_{1}\right\}$ be a closed segment of length $2 d_{1}$ centered around $V$. This is shown in Figure 4 . The distance from the sender at $X_{i}$ to any point $U$ of the segment $\mathcal{Z}$ satisfies $^{4} d\left(X_{i}, U\right)<2 d_{1}$. It follows that if the receiver $X_{j(i)} \in \mathcal{Z}$, then $P_{i} \geq\left(2 d_{1}\right)^{-\alpha}=\left[2 \sin ^{-1}\left(\frac{1}{\gamma \sqrt{n}}\right)\right]^{-\alpha}$. Note that

$$
\lim _{n \rightarrow \infty}\left[\sqrt{n} 2 \sin ^{-1}\left(\frac{1}{\gamma \sqrt{n}}\right)\right]^{-\alpha}=\left[\frac{\gamma}{2}\right]^{\alpha}
$$

Therefore, asymptotically in $n$, if a receiver node whose great circle intersects $D_{X_{i}, d_{1}}$ and is within the segment $\mathcal{Z}$, then it receives at a power $\Theta\left(n^{\alpha / 2}\left[\frac{\gamma}{2}\right]^{\alpha}\right)$. We now bound the asymptotic probability that there is at least one receiver node sufficiently close to $X_{i}$. Assume that $B \leq\left(\frac{\gamma}{2}\right)^{\alpha}$. Then,

$$
\begin{aligned}
\lim \inf _{n \rightarrow \infty} \operatorname{Pr}\left\{n^{-\alpha / 2} P_{i}>B \mid X_{i}\right\} & \geq \lim \inf _{n \rightarrow \infty}\left[1-\operatorname{Pr}\left\{X_{j} \notin \mathcal{Z}_{j},\left\{G_{j} \cap D_{X_{i}, d_{1}} \neq \phi\right\} \cap \mathcal{R}_{t}\right\}\right] \\
& =\lim \inf _{n \rightarrow \infty}\left[1-\left(\operatorname{Pr}\left\{X_{j} \notin \mathcal{Z}_{j}\right\}\right)^{N_{1}^{\prime}}\right]
\end{aligned}
$$

where $N_{1}^{\prime}=\left|\left\{G_{j} \cap D_{X_{i}, d_{1}} \neq \phi\right\} \cap \mathcal{R}_{t}\right|$ is the number of great circles intersecting the disk $D_{X_{i}, d_{1}}$ that are potential receivers at time $t$ and $\mathcal{Z}_{j}=\left\{U \in G_{j}: d(U, V) \leq d_{1}\right\}$. Now $N_{1}^{\prime}=\Theta(\sqrt{n})$ with high probability, because $\left|\left\{G_{j} \cap D_{X_{i}, d_{1}} \neq \phi\right\}\right|=N_{1}=\Theta(\sqrt{n})$, and because potential receivers are selected randomly at every time slot $t$; therefore, $N_{1}^{\prime} \rightarrow N_{1} / 2$ a.s. Here we have used the limit specified in (20) to justify the inequality used.

$$
\lim _{n \rightarrow \infty}\left(\operatorname{Pr}\left\{X_{j} \notin \mathcal{Z}_{j}\right\}\right)^{N_{1}^{\prime}} \leq \lim _{n \rightarrow \infty}\left(1-\frac{d_{1}}{\pi}\right)^{\sqrt{n} / 2 \gamma} \stackrel{(a)}{=} \lim _{n \rightarrow \infty}\left(1-\frac{1}{\pi \gamma \sqrt{n}}\right)^{\sqrt{n} / 2 \gamma}=e^{-\frac{1}{2 \gamma^{2} \pi}}<1,
$$

where in $(a)$ we have used the limit argument of (20). Therefore, $c_{P}>0$, for any constant $B$ since we can choose an appropriate $\gamma$ for any given $B$.

\footnotetext{
${ }^{4}$ The inequality is because the distance metric is defined as distance on the surface of the sphere. Hence, $\cos \left[d\left(X_{i}, U\right)\right]=\cos \left[d\left(X_{i}, V\right)\right] \cos [d(V, U)]$ by using the geometry of the triangle formed by $\left\{X_{i}, U, V\right\}$ on the surface of the sphere [5]. Moreover, for distances smaller than $\pi / 2$, it can be shown that the sum of two sides of a triangle on the sphere is less than the third (analogous to the result of triangles on a plane). This can also be seen by noticing that for angles smaller than $\pi / 2, \cos \left[d\left(X_{i}, U\right)\right]=\cos \left[d\left(X_{i}, V\right)\right] \cos [d(V, U)] \geq \cos \left[d\left(X_{i}, V\right)+d(V, U)\right]$. Since we are dealing with $n$ large, this condition is clearly satisfied. Hence, given that $d(V, U) \leq d_{1}$ and $d\left(X_{i}, V\right) \leq d_{1}$, this shows that $d\left(X_{i}, U\right) \leq 2 d_{1}$.
} 


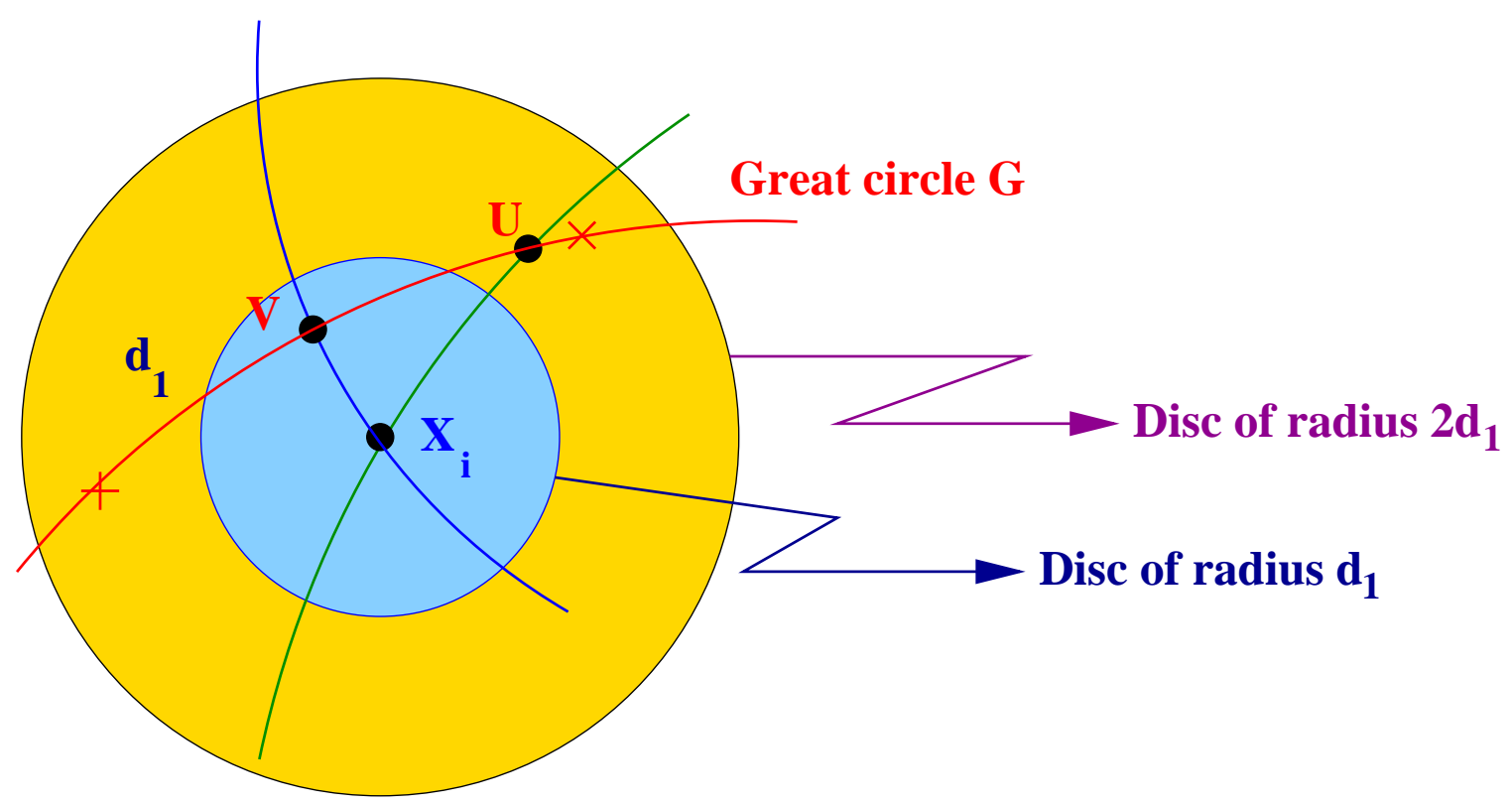

Figure 4: Disc of size $d_{1}$ around sender $X_{i}$ with great circle $G$ intersecting disc used in proof of Lemma 4.5.

\section{B Proof of Lemma 4.6}

Proof: We analyze the conditional interference power $I_{i}$, conditional on the location $X_{j}$ of receiver $j(i)$ for a typical constellation. We can upper bound $I_{i}$ by lower bounding the distance of the receiver $j$ from an interfering sender $k$.

We use the following fact: the received power $P_{k j}$ at $X_{j}$ from node $k$ (on great circle $G_{k}$ ) is stochastically upper bounded by another node $k^{\prime}$ (on a great circle $G_{k^{\prime}}$ ) that satisfies $d_{\mathbb{S}}\left(X_{j}, G_{k^{\prime}}\right) \leq$ $d_{\mathbb{S}}\left(X_{j}, G_{k}\right)^{5}$. Also, we make the conservative assumption that all nodes are senders, instead of just the nodes in $\mathcal{S}_{t}$.

Let the receiver location be denoted by $X_{j} \in \mathbb{S}$. First, we draw disks $D_{X_{j}, d_{l}}$ centered around the receiver position, where we choose radii $d_{l}$ such that $\sin \left(d_{l}\right)=\frac{l}{\gamma \sqrt{n}}$. This is illustrated in Figure 6. Let $W_{l}^{\prime}$ denote the total received interference power from all the great circles in the annulus $D_{X_{j}, d_{l}} \backslash D_{X_{j}, d_{l-1}}$. This is stochastically upper bounded by considering that all the $N_{l}$ great circles in this annulus are at a distance $d_{l-1}$ from the receiver. We denote the power from the $m=1, \ldots, N_{l}$ nodes at a distance $d_{l-1}$ by $W_{l, m}$. Therefore, we can stochastically upper bound $I_{i}$ with

$$
I_{i} \stackrel{s t}{\leq} \sum_{l=1}^{\gamma \sqrt{n}} \sum_{m=1}^{N_{l}} W_{l, m} .
$$

Note that all the $W_{l, m}$ random variables are independent. We notice that by using (9), for a typical constellation we obtain $N_{l}=\Theta(\sqrt{n})$.

\footnotetext{
${ }^{5}$ In fact, this relationship only holds on the hemisphere with $X_{j}$ as its pole; the inequality is inverted on the "far" hemisphere. However, it is easy to show that this does not affect our result, because the impact of the "far" nodes is negligible.
} 
Let us first focus on $W_{l, m}$ for $l \geq 2$. For these random variables, there is a lower bound on the distance from the receiver point $X_{j}$ of $d_{l}=\sin ^{-1}\left(\frac{l-1}{\gamma \sqrt{n}}\right)$. Let us examine a great circle $G$ at distance $d_{l}$ from the receiver point $X_{j}$, and let the closest point on $\mathrm{G}$ from receiver point $X_{j}$ (see Figure 5) be denoted by $A$ on the great circle $G$. Let the node travel on the great circle and be at distance of $z$ from the point $A$ of the great circle. If the distance of node from the receiver point is denoted as $v$, then the following inequality can be shown,

$$
\begin{aligned}
& \cos (v)=\cos \left(d_{l}\right) \cos (z) \\
& \Longrightarrow|v| \geq\left\{\begin{array}{cc}
\max \left\{\left|d_{l}\right|,|z|\right\}, & \text { for }|z| \leq \pi / 2 \\
\pi / 2, & |z| \geq \pi / 2
\end{array}\right.
\end{aligned}
$$

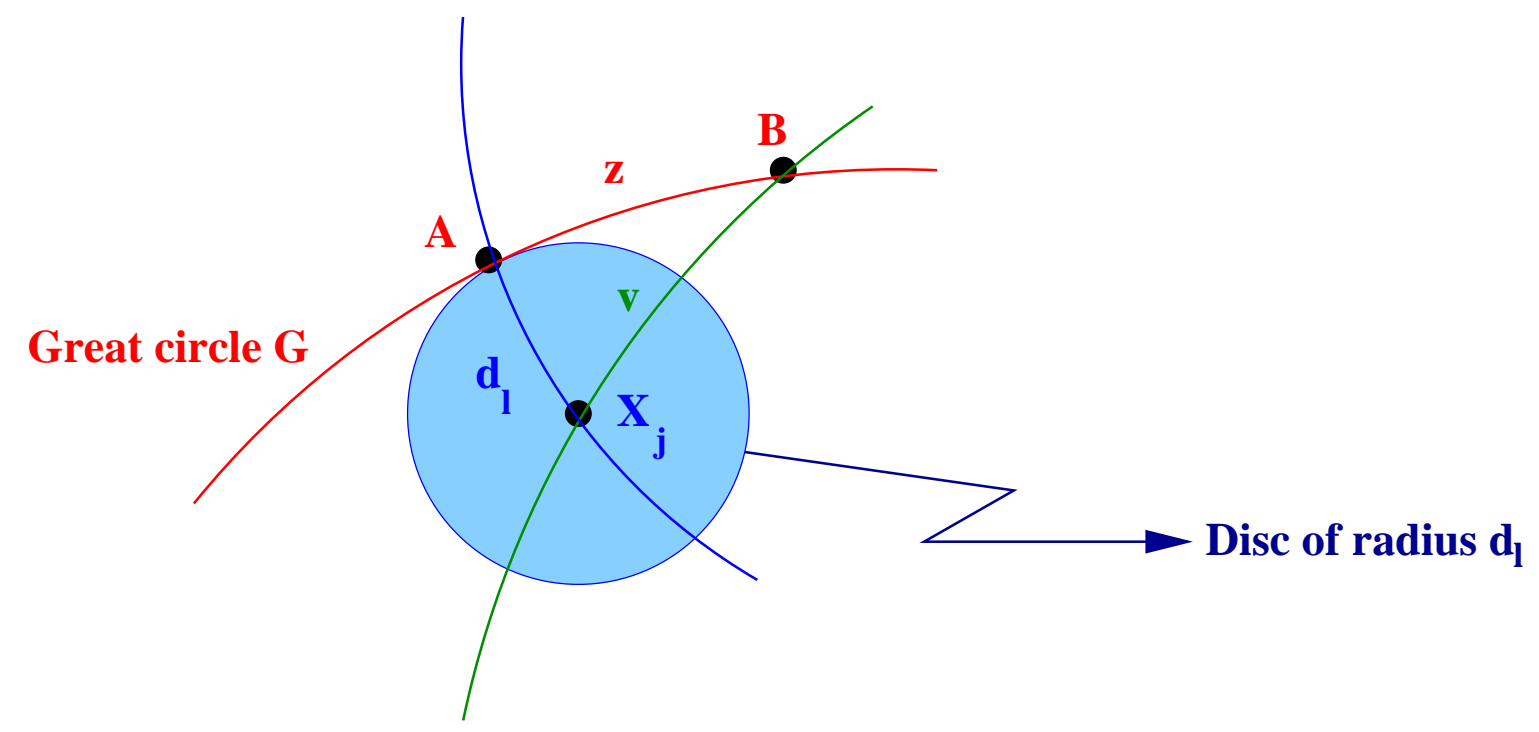

Figure 5: Figure illustrating bound on power of interferer on great circle at a distance $d_{l}$ from receiver $X_{j}$ used in proof of Lemma 4.6.

If the node mobility is stationary and ergodic on the great circle (GC), then the empirical probability distribution of the node on the $\mathrm{GC}$ is also uniform, i.e., $Z \sim \operatorname{unif}(-\pi, \pi)$. Using this and (24) we obtain,

$$
\begin{aligned}
\mathrm{E}\left[\frac{1}{v^{\alpha}}\right] & \leq \frac{2}{\pi} \int_{z=0}^{z=d_{l}} \frac{1}{\max \left\{d_{l}^{\alpha}, z^{\alpha}\right\}} d z+\frac{2}{\pi} \int_{z=d_{l}}^{z=\pi / 2} \frac{1}{\max \left\{d_{l}^{\alpha}, z^{\alpha}\right\}} d z+\frac{2}{\pi} \int_{z=\pi / 2}^{z=\pi} \frac{1}{(2 / \pi)^{\alpha}} d z \\
& =\frac{2}{\pi} \int_{z=0}^{z=d_{l}} \frac{1}{d_{l}^{\alpha}} d z+\frac{2}{\pi} \int_{z=d_{l}}^{z=\pi / 2} \frac{1}{z^{\alpha}} d z+\left(\frac{2}{\pi}\right)^{\alpha} \\
& =\frac{2 \alpha}{\pi(\alpha-1)} \frac{1}{d_{l}^{\alpha-1}}+\frac{\alpha-2}{\alpha-1}\left(\frac{2}{\pi}\right)^{\alpha} \\
& \leq \frac{2 \alpha}{\pi(\alpha-1)} \frac{1}{\sin ^{\alpha-1}\left(d_{l}\right)}+\frac{\alpha-2}{\alpha-1}\left(\frac{2}{\pi}\right)^{\alpha}
\end{aligned}
$$


To find the probability of large interference, we can write

$$
\begin{aligned}
\operatorname{Pr}\left\{n^{-\alpha / 2} I_{i}>A \mid X_{j}\right\} & \leq \operatorname{Pr}\left\{n^{-\alpha / 2} \sum_{l=1}^{\gamma \sqrt{n}} \sum_{m=1}^{N_{l}} W_{l, m}>A \mid X_{j}\right\} \\
& \leq \operatorname{Pr}\left\{n^{-\alpha / 2} \sum_{m=1}^{N_{1}} W_{1, m}>\frac{A}{2} \mid X_{j}\right\}+\operatorname{Pr}\left\{n^{-\alpha / 2} \sum_{l=2}^{\gamma \sqrt{n}} \sum_{j=1}^{N_{l}} W_{l, m}>\frac{A}{2} \mid X_{j}\right\}
\end{aligned}
$$

We need to show that $A$ can be made large enough so that $(26)$ is less than a constant $c_{I}<1$. For the first term, note that the great circles passing through the innermost disk would cause an infinite mean power; nevertheless, we can use the theory of stable distributions to bound this term. For the second term, we use the inequalities developed in (25), and show that for a large enough $A$ this probability can be made small enough.

Innermost disk: $\operatorname{Pr}\left\{n^{-\alpha / 2} \sum_{m=1}^{N_{1}} W_{1, m}>A / 2 \mid X_{j}\right\}$. We can conservatively assume that these nodes lie on the same great circle as the receiver. Therefore, the $W_{1, m}$ are i.i.d. with distribution $\operatorname{Pr}\left\{W_{1, m} \geq y\right\}=\frac{\delta}{y^{1 / \alpha}}$ for $y \geq \delta^{\alpha}$, where $\delta=\frac{1}{\pi}$, and one elsewhere. This can be seen since $\operatorname{Pr}\left\{W_{1, m} \geq y\right\}=\operatorname{Pr}\left\{z_{m}^{-\alpha} \geq y\right\}$, where $z_{m}$ is the distance of interferer to the receiver; using the uniform distribution of the location of the interferer on the great circle, we get the stated result. Note $z_{m} \leq \pi$, yielding $W_{1, m} \geq \pi^{-\alpha}=\delta^{\alpha}$.

Now note that the distribution of $W_{1, m}$ satisfies the requirement for $M^{-\alpha} \sum_{m=1}^{M} W_{1, m}$ to converge to a stable distribution with characteristic exponent $\frac{1}{\alpha}$ (see [2], pp 448, Theorem 2). Since $N_{1}=\Theta(\sqrt{n})$ by the typicality of constellations, $N_{1}^{-\alpha} \sum_{m=1}^{N_{1}} W_{1, m}=C^{-\alpha / 2} n^{-\alpha / 2} \sum_{m=1}^{N_{1}} W_{1, m}$, for some constant $C$. Hence it follows that $\operatorname{Pr}\left\{n^{-\alpha / 2} \sum_{m=1}^{N_{1}} W_{1, m}>A / 2 \mid X_{j}\right\}$ can be made arbitrarily small by choosing $A$ large enough. Note that we need to make it small enough so that the terms in (26) sum to less than 1 , and such a choice would depend on the bound on $\operatorname{Pr}\left\{n^{-\alpha / 2} \sum_{l=2}^{\gamma \sqrt{n}} \sum_{j=1}^{N_{l}} W_{l, m}>\frac{A}{2} \mid X_{j}\right\}$ which is given below.

Outer disks: $\operatorname{Pr}\left\{n^{-\alpha / 2} \sum_{l=2}^{\gamma \sqrt{n}} \sum_{m=1}^{N_{l}} W_{l, m}>A / 2 \mid X_{j}\right\}$. For this we just use Markov's inequality, i.e., $\operatorname{Pr}\{X>a\} \leq \mathrm{E}[X] / a$. We obtain,

$$
\begin{aligned}
\operatorname{Pr}\left\{n^{-\alpha / 2} \sum_{l=2}^{\gamma \sqrt{n}} \sum_{m=1}^{N_{l}} W_{l, m}>A / 2 \mid X_{j}\right\} & \leq \sum_{l=2}^{\gamma \sqrt{n}} \sum_{m=1}^{N_{l}} \frac{\mathrm{E}\left[W_{l, m}\right]}{A n^{\alpha / 2} / 2}=\sum_{l=2}^{\gamma \sqrt{n}} N_{l} \frac{\mathrm{E}\left[W_{l, m}\right]}{A n^{\alpha / 2} / 2} \\
& \leq \sum_{l=2}^{\gamma \sqrt{n}} \frac{2 N_{l}}{A n^{\alpha / 2}}\left[\frac{2 \alpha}{\pi(\alpha-1)} \frac{1}{\sin ^{\alpha-1}\left(d_{l}\right)}+\frac{\alpha-2}{\alpha-1}\left(\frac{2}{\pi}\right)^{\alpha}\right] \\
& \leq \sum_{l=2}^{\gamma \sqrt{n}} \frac{2 N_{l}}{A n^{\alpha / 2}}\left[\frac{2 \alpha}{\pi(\alpha-1)} \frac{(\gamma \sqrt{n})^{\alpha-1}}{(l-1)^{\alpha-1}}+\frac{\alpha-2}{\alpha-1}\left(\frac{2}{\pi}\right)^{\alpha}\right] \\
& \leq \frac{c}{A} \sum_{l=2}^{\gamma \sqrt{n}} \frac{1}{(l-1)^{\alpha-1}}+o(1),
\end{aligned}
$$


where $c>0$ is a constant. The sum converges for $\alpha>2$, which establishes that we can choose $A$ to make the second term arbitrarily small as well. Therefore we can choose $A$ large enough to make the sum of the terms in (26) less than 1. This completes the proof of Lemma 4.6.

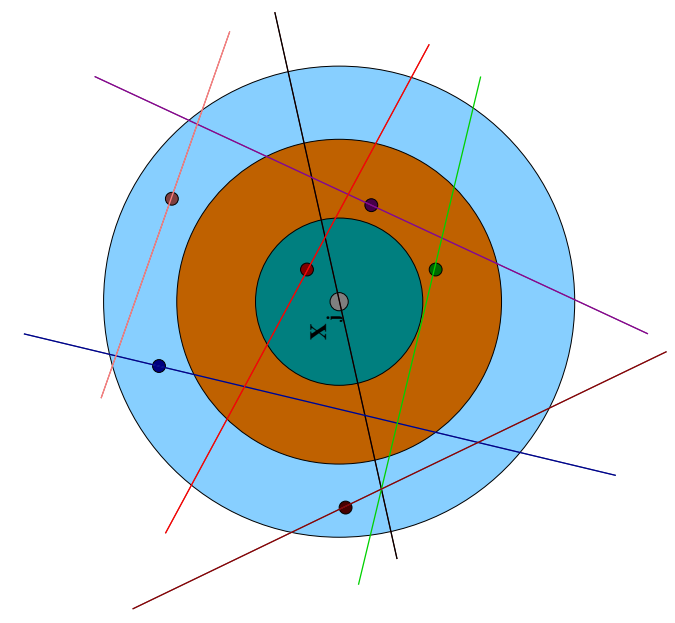

Figure 6: Binning of region around receiver $X_{j}$ with discs used in proof of Lemma 4.6.

\section{References}

[1] Luc Devroye, Laszlo Gyorfi, and Gabor Lugosi. A Probabilistic Theory of Pattern Recognition. Springer Verlag, New York, 1996.

[2] William Feller. An introduction to probability theory and its applications: Part II. Wiley, New York, 1968.

[3] M. Grossglauser and D. N. C. Tse. Mobility Increases the Capacity of Ad-hoc Wireless Networks. IEEE/ACM Transactions on Networking, 2002.

[4] Piyush Gupta and P. R. Kumar. The Capacity of Wireless Networks. IEEE Transactions on Information Theory, 46(2):388-404, March 2000.

[5] J. Stillwell. Geometry of surfaces. Springer-Verlag Publishers, New York, 1992.

[6] David Williams. Probability with Martingales. Cambridge University Press, New York, 1991. 